\title{
Nanoscale
}

\section{Facile multifunctional plasmonic sunlight harvesting with tapered triangle nanopatterning of thin films $\uparrow$}

\author{
Giulia Tagliabue, Hadi Eghlidi* and Dimos Poulikakos* \\ Plasmonic absorbers have recently become important for a broad spectrum of sunlight-harvesting \\ applications exploiting either heat generation, such as in thermal photovoltaics and solar \\ thermoelectrics, or hot-electron generation, such as in photochemical and solid state devices. So far, \\ despite impressive progress, combining the needed high performance with fabrication simplicity and \\ scalability remains a serious challenge. Here, we report on a novel solar absorber concept, where we \\ demonstrate and exploit simultaneously a host of absorption phenomena in tapered triangle arrays \\ integrated in a metal-insulator-metal configuration to achieve ultrabroadband (88\% average \\ absorption in the range of 380-980 nm), wide-angle and polarization-insensitive absorption. \\ Furthermore, this absorber is subwavelength in thickness $(260 \mathrm{~nm})$ and its fabrication is based on a \\ facile, low-cost and potentially scalable method. In addition, the geometry of our design makes it \\ compatible for both heat and hot electron generation.
}

Received 25th June 2013 Accepted 30th July 2013

DOI: $10.1039 / c 3 n r 03273 f$

www.rsc.org/nanoscale
While different geometries showed further improvements ${ }^{\mathbf{1 5}}$ their small scale structuring is attained with difficulty and the upscaling of fabrication methods seems improbable. A different theoretical approach based on conformal transformations has exploited non-resonant nano-focusing at the end of sharp tips or grooves to achieve plasmonic nanostructures with very large absorption cross-sections over a broad spectral range. ${ }^{\mathbf{1 6 - 1 8}}$ Following a similar principle, Sondergaard et al. ${ }^{\mathbf{1 9 , 2 0}}$ realized a broadband absorber consisting of an array of ultra-sharp convex grooves. Despite impressive results (>87\% absorption over $450-$ $850 \mathrm{~nm}$ ) their design requires extremely narrow features, which are achieved by a cumbersome combination of electron-beam lithography and focused ion-beam milling (also practically impossible to upscale). Moreover, the structure is not subwavelength in thickness and the focusing hot-spots are deeply buried into the structure, therefore inaccessible for several light harvesting applications. In this paper, we address these issues by a concept, which exploits various plasmonic absorption phenomena in an array of tapered triangles within an MIM structure. While achieving a near-ideal solar absorber, we are able to fabricate the device with a potentially low-cost and easily accessible method, a must if plasmonic solar harvesting is to become a reality outside the research laboratory.

\section{Results and discussion}

Technology, Department of Mechanical and Process Engineering, ETH Zürich, CH-8092 Zürich, Switzerland. E-mail: hadi.eghlidi@ltnt.iet.mavt.ethz.ch; dpoulikakos@ethz.ch; Fax:+41 4463211 76; Tel: +41 4463206 77; +41 446322738 $\dagger$ Electronic supplementary information (ESI) available: Detailed geometry description; set-up description; literature comparison; surface plasmon polariton dispersion curve; absorption efficiency of an isolated tapered triangle on glass. See DOI: 10.1039/c3nr03273f
The schematic representation of the absorber is given in Fig. 1a and $b$, where all the relevant geometrical parameters are introduced. The MIM structure is composed of three layers (see Fig. 1a). A $100 \mathrm{~nm}$ gold back-reflector and a $60 \mathrm{~nm} \mathrm{SiO}{ }_{2}$ layer were coated successively on a glass coverslip using electron-beam 
evaporation and plasma-enhanced chemical vapor deposition, respectively. A front hexagonal gold pattern was fabricated using nano-sphere lithography (NSL) ${ }^{21-24}$ and reactive ion etching (RIE). $\$$ The schematic top-view of the front pattern is shown in Fig. 1b. $R$ is defined as the radius of curvature of the pattern, and $R_{\text {bead }}$ is the radius of the spheres (beads) which are used in the NSL and constitute a closely packed hexagonal array. $\Delta R$ is defined as the difference between $R$ and $R_{\text {bead }}$. For $\Delta R>0$, the front pattern consists of an array of disconnected gold triangles with curved sides. The neighbouring tapered triangles touch each other at a single point when $\Delta R=0$. Due to overshadowing, ${ }^{24}$ experimentally $\Delta R>0$ can be obtained and fine-adjusted by varying the etching time of the RIE process mentioned above. By doing this we can vary the size of the triangles and the tapering of their tips ( $2 \theta$, see Fig. 1b). A top view scanning electron micrograph of a created structure is shown in Fig. 1c.

The performance of the proposed absorber is illustrated in Fig. $2 b-j$, where the measured and calculated absorption

\$ Fabrication procedure details. Clean glass coverslips $(18 \times 18 \mathrm{~mm})$ were coated first with a $100 \mathrm{~nm}$ gold back reflector (electron beam evaporation) and successively with a $60 \mathrm{~nm} \mathrm{SiO}_{2}$ layer (plasma enhanced chemical vapor deposition). The thickness of the dielectric spacer and optical properties of $\mathrm{SiO}_{2}$ and gold layers were always measured using ellipsometry. After cleaning with a $6: 1: 1$ solution of $\mathrm{H}_{2} \mathrm{O}: \mathrm{NH}_{4} \mathrm{OH}: \mathrm{H}_{2} \mathrm{O}_{2}$ in an ultrasonic bath for $10 \mathrm{~min}$, the samples were stored for $24 \mathrm{~h}$ in ethanol to ensure full hydrophilicity of the glass surface. The hexagonal gold patterns are fabricated using nanosphere lithography (NSL) and etching techniques. First, a closely packed monolayer of polystyrene beads (Sigma Aldrich, diameter $300 \mathrm{~nm}$ ) is created using dip-coating (see ESI S2 $\uparrow$ for the set-up): the sample is quickly immersed into a diluted bead solution ( $1 \mathrm{wt} \%, 10^{-3} \mathrm{M}$ SDS) and then withdrawn with controlled velocity $\left(\approx 2.5 \mu \mathrm{m} \mathrm{s}^{-1}\right)$. The humidity is kept constant at $\approx 50 \%$. Then, reactive ion etching is used to reduce the bead size (100 $\mathrm{sccm} \mathrm{Ar}, 10 \mathrm{sccm} \mathrm{O}_{2}, 50 \mathrm{~W}, 100 \mu \mathrm{bar}$ ). The bead size is controlled by adjusting the etching time. Due to overshadowing in the NSL, ${ }^{24}$ zero or short etching times result in disconnected curved triangles $(\Delta R>0)$. The final pattern is created by evaporating $100 \mathrm{~nm}$ of gold (after $2 \mathrm{~nm}$ Cr adhesion layer) through the bead mask and by successively removing the beads by low power ultrasonication.

Optical Measurements. Absorption measurements are performed using a home-built inverted microscope equipped with an air objective (numerical aperture 0.75) for exciting the sample and collecting the reflected and scattered light and a spectrometer (Princeton Instruments). White light is generated by a xenon lamp and is brought to the set-up with an optical fiber (105 $\mu \mathrm{m}$ core size) in order to improve its spatial coherence. Plane wave excitations with different angles of incidence are achieved by focusing the light beam at different positions on the back focal plane of the objective. The relation $\mathbf{A}=(\mathbf{I}-\mathbf{R}) / \mathbf{I}$ was used to calculate the absorption spectrum, where $\mathbf{A}$, $\mathbf{I}$ and $\mathbf{R}$ are the absorption, incident (excitation) and reflected spectra, respectively. The light transmitted through the absorber was negligible. I was measured using the reflected spectrum from a broadband silver mirror. This ensures accounting for excitation spectral non-uniformity and loss along the detection path. Furthermore, dark counts of the spectrometer and background spectra were subtracted from all the measured spectra.

Simulations. Primary estimation of optimal dimensions was achieved by performing a large series of full-wave simulations with Lumerical, a commercially available finite-difference time-domain (FDTD) software package, which is very efficient for broadband simulations. However, due to the staircasing errors of FDTD at sharp edges, the final simulations (which include all the results shown in this manuscript) were performed using the COMSOL Multiphysics package (RF module), which is based on the finite element method (FEM). By using symmetry and periodic (for normal excitation) or Floquet (for oblique excitation) boundary conditions the computational domain was reduced to one-fourth of a unit-cell. To model gold in the simulations, we used the optical properties tabulated in Palik's Handbook ${ }^{25}$ which agree well with our ellipsometry measurements (see Fabrication procedure details). (a)

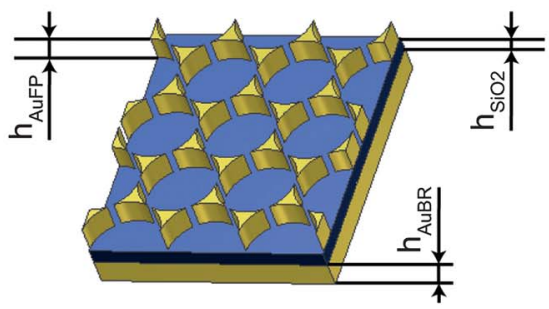

(b)

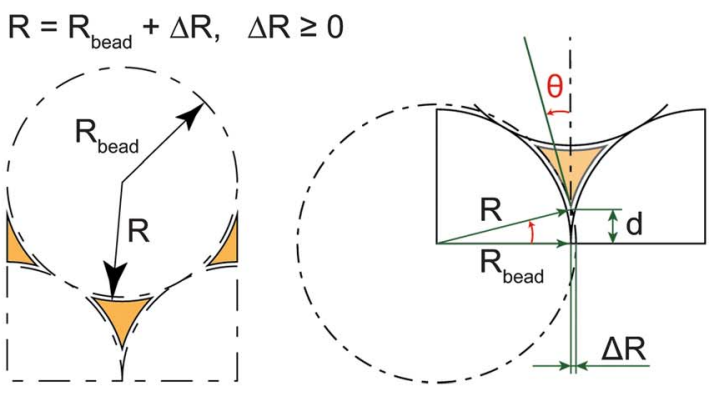

(c)

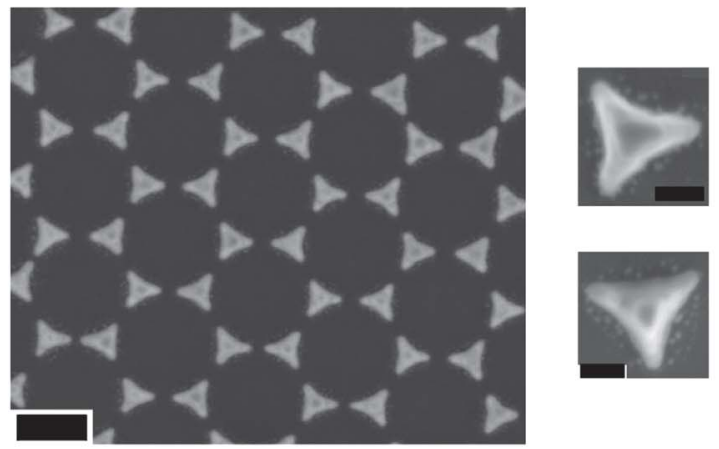

Fig. 1 Geometry of the proposed absorber. (a) Schematic representation of the MIM absorber consisting of a gold back reflector, a $\mathrm{SiO}_{2}$ dielectric spacer, and a patterned gold front layer with thicknesses $h_{\mathrm{AuBR},} h_{\mathrm{SiO}_{2},}$ and $h_{\text {AuFP, }}$ respectively. (b) Definition of the in-plane geometrical parameters of the front gold pattern: $R_{\text {bead }}$ is the radius of the spherical beads used in the NSL, and $R$ is defined as the radius of curvature of the pattern. $\Delta R$ represents the difference between $R$ and $R_{\text {bead }}$ and can be adjusted in the fabrication. $2 \theta$ and $2 d$ are the tip tapering angle and the distance between two neighboring tips, respectively. (c) Left: top-view scanning electron micrograph of a fabricated structure. Scale bar is $200 \mathrm{~nm}$. Right: magnified top views of single tapered triangles. Scale bars are $50 \mathrm{~nm}$.

spectra are plotted for different values of $\Delta R$. The other geometrical parameters are fixed at $R_{\text {bead }}=150 \mathrm{~nm}$ and $h_{\mathrm{SiO}_{2}}=$ $60 \mathrm{~nm}$ (see Fig. 2a), and the thickness of the back reflector and the gold triangles are both equal to $100 \mathrm{~nm}$. The overall structure is only $260 \mathrm{~nm}$ thick. The numerical simulations are carried out using the COMSOL Multiphysics package (RF Module) $\$$ and the absorption measurements were performed using a home-built inverted microscope. Collimated white light was used for excitation and the absorption spectrum, A, was calculated as $\mathbf{A}=(\mathbf{I}-\mathbf{R}) / \mathbf{I}$, where $\mathbf{I}$ and $\mathbf{R}$ are the incident (excitation) and reflected spectra, respectively. The light transmitted through the absorber was negligible.

Both simulation and experimental data suggest that decreasing $\Delta R$ (equivalently decreasing 2 $\theta$ ) increases the 
absorption bandwidth while maintaining reasonably high absorption within the bandwidth. The best experimental result in terms of broadness is obtained at $\Delta R \approx 2 \mathrm{~nm}$ (see Fig. $2 \mathrm{j}$ ), for which the neighbouring tapered triangles' tips are at the closest distance but still separated. Here, absorption stays above $85 \%$ within the spectral range of $380-850 \mathrm{~nm}$ and slowly drops to $70 \%$ at $980 \mathrm{~nm}$. We reach an average absorption slightly higher than $88 \%$ within the spectral range of $380-980 \mathrm{~nm}$. The numerical results in Fig. $2 \mathrm{~b}$ show a rapid increase in the absorption bandwidth as $\Delta R$ approaches zero (equivalently, the distance between the tips of neighbouring triangles as well as $\theta$ decrease). As mentioned above, $\Delta R$ can be controlled with nanometer resolution by using a slow enough etching process. While the fabrication relies on a facile method, the results show the potential of the proposed concept to easily adjust the absorption bandwidth and achieve a near-ideal absorber.

Angular insensitivity is one of the important properties of an ideal absorber. To assess the angular sensitivity of our absorber we illuminated it with plane waves at different angles of incidence. We obtained this by focusing the excitation light at different positions on the back-focal plane of the objective with which we were able to reach angles up to 48 degrees (numerical aperture, $\mathrm{NA}=0.75)$. The measured absorption spectra for the best experimental case (Fig. 2j) for incidence angles from 0 to 45

(a)

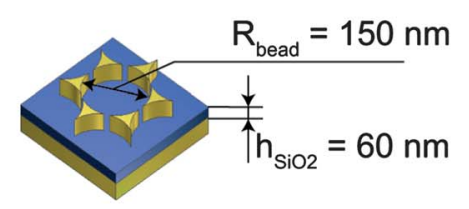

(b)

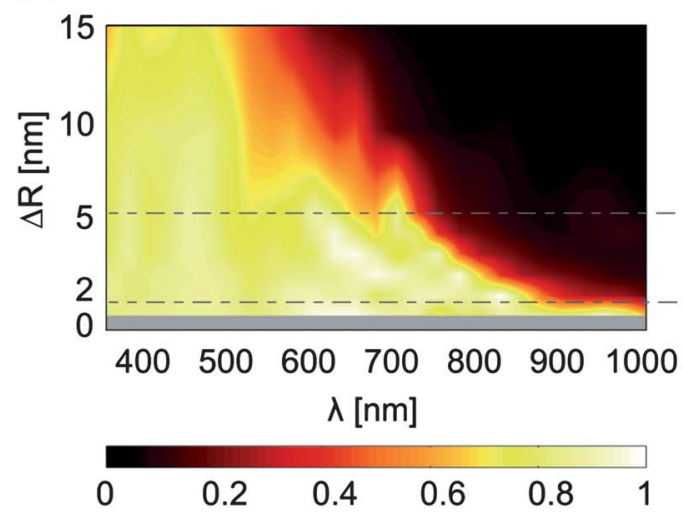

(k)
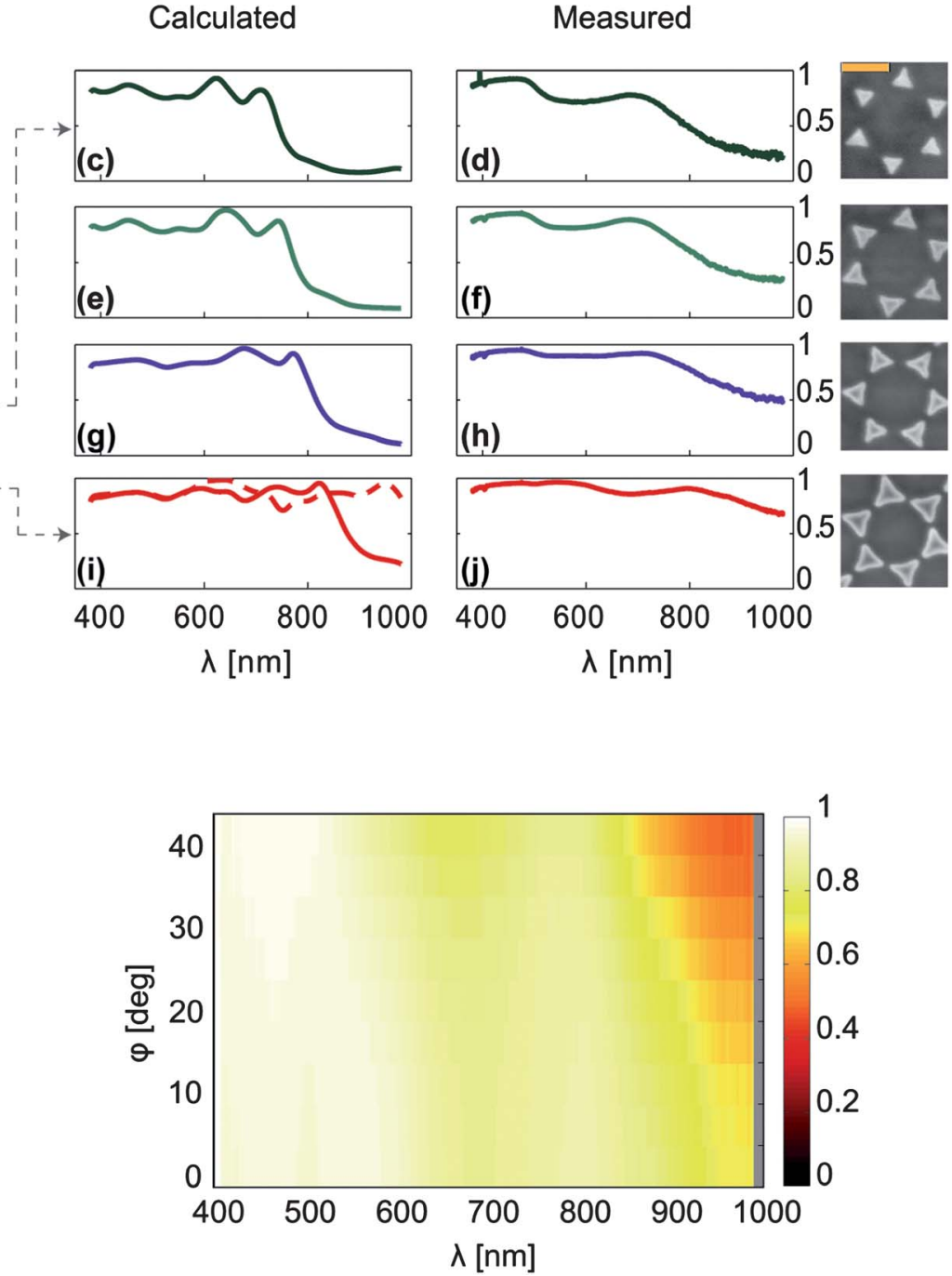

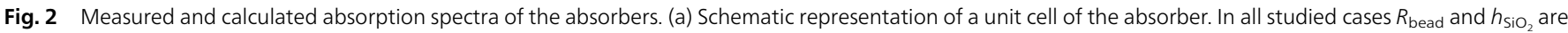

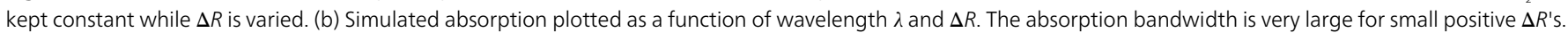

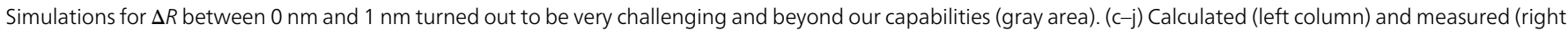

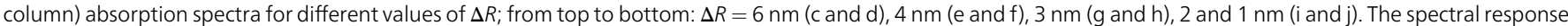

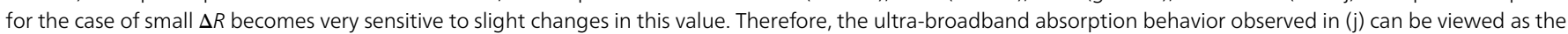

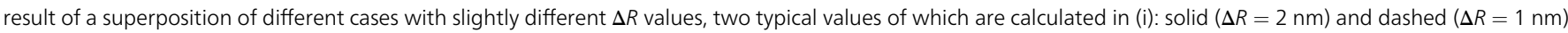

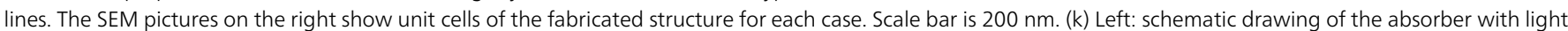

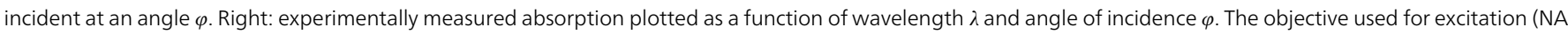
$=0.75$ ) allowed excitation angles up to $\varphi=48^{\circ}$. 
degrees are summarized in Fig. 2k. The results show that a broad absorption spectrum is preserved even at large angles of incidence. ${ }^{26}$ Finally, our design concept is inherently polarization insensitive at normal incidence. This is due to the geometrical planar symmetries that it contains. In addition, the measurements, which are performed with unpolarized light, and the simulations (not shown for brevity) confirm a similar insensitivity for angles away from the normal.

The broadband absorption behaviour of our design is the collective result of a number of plasmonic absorption events. These events occur at different locations of the spectrum and, after appropriate choice of the geometrical dimensions, piece together a continuous absorption band. To assess the impact of the dielectric spacer thickness, in Fig. 3 a we show the calculated absorption spectra for different values of $h_{\mathrm{SiO}_{2}}$. All of the other geometrical parameters are kept constant $\left(R_{\text {bead }}=150 \mathrm{~nm}\right.$, $h_{\mathrm{Au}, \mathrm{FP}}=100 \mathrm{~nm}, \Delta R=2 \mathrm{~nm}$, see Fig. $2 \mathrm{a}$ ). The horizontal dashed line shows the situation that we have in the experiment, $h_{\mathrm{SiO}_{2}}=$ $60 \mathrm{~nm}$ (Fig. 2j), which leads to very high absorption over the entire absorption spectrum. Moving away from this optimal thickness leads to the appearance of spectral bands with high reflection in the visible range. To understand the origin of the broadband absorption, we studied the field distributions inside the dielectric layer on two horizontal cross-sections, close to the top pattern and close to the back reflector. Fig. 3d shows magnetic field enhancement profiles at four absorption peaks indicated by $\mathrm{P}_{1}-\mathrm{P}_{4}$ in Fig. 3a.

At $\mathrm{P}_{1}(\lambda=470 \mathrm{~nm})$ we note that the electromagnetic energy is concentrated close to the back-reflector. This indicates that the absorption at this wavelength originates from the gratingmediated coupling of electromagnetic field to the surface plasmon polariton (SPP) excited at the interface between the $\mathrm{SiO}_{2}$ layer and the gold back reflector. ${ }^{27}$ Our analytical calculations for the simplified three-layer (air-SiO ${ }_{2}$-gold) structure predict the grating coupled SPP (corresponding to the grating period $=2 R_{\text {bead }}=300 \mathrm{~nm}$ ) at $\lambda=498 \mathrm{~nm}$ (see ESI, Fig. S $2 \dagger$ ), which is close to the observed resonance. This absorption resonance only slightly blue-shifts as the value of $h_{\mathrm{SiO}_{2}}$ (a)

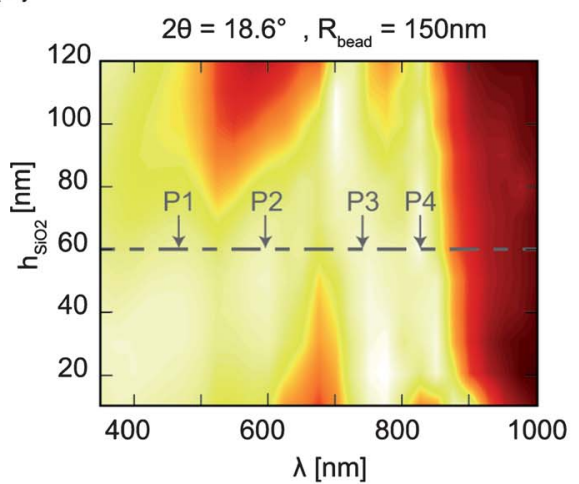

(b)

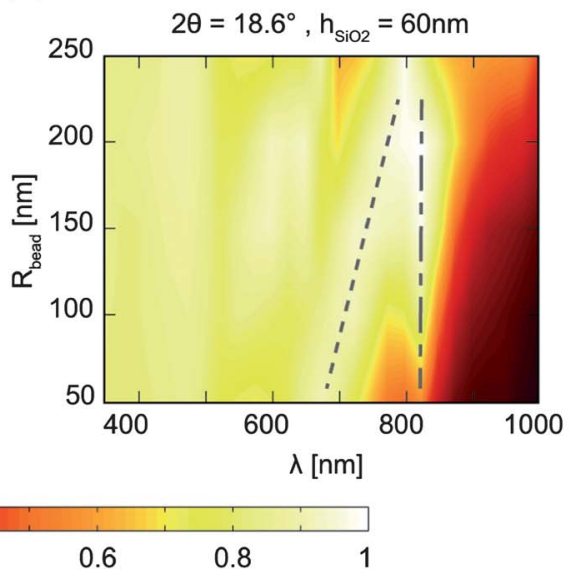

(c)

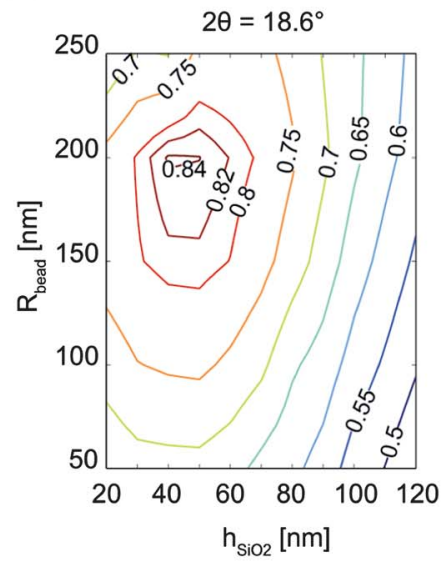

(d)

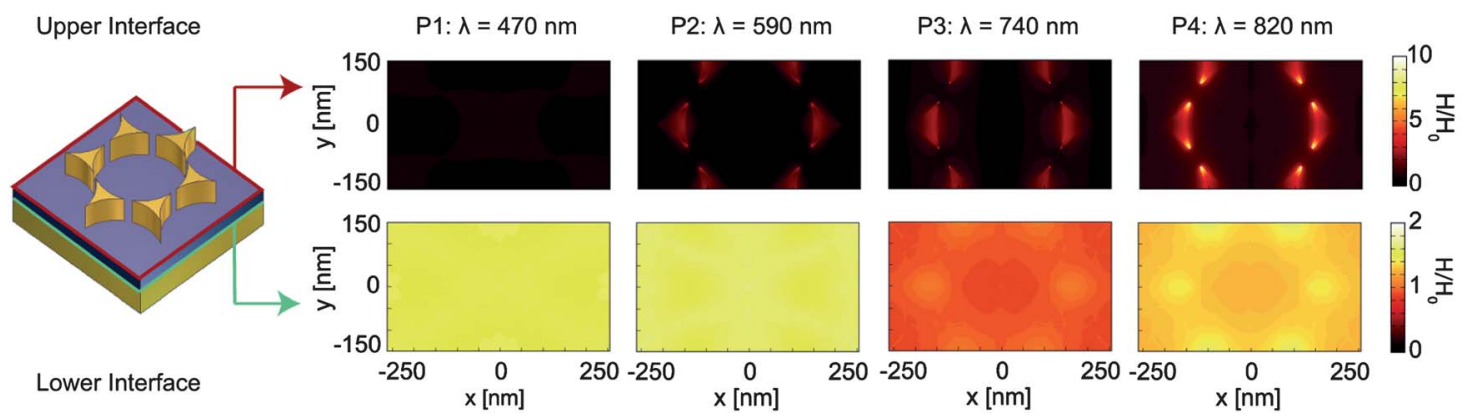

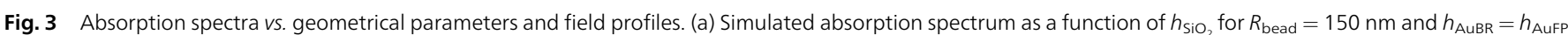

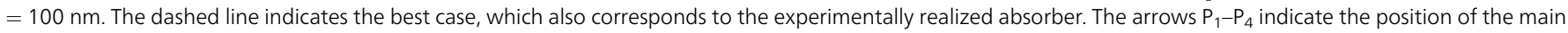

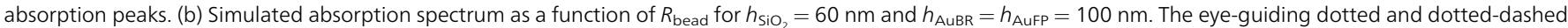

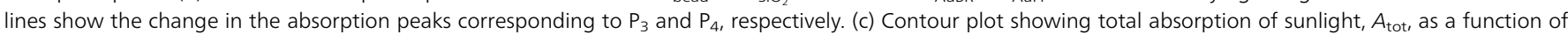

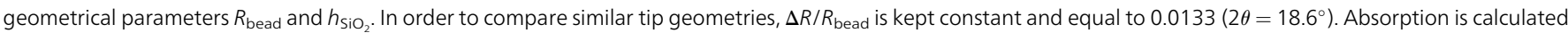

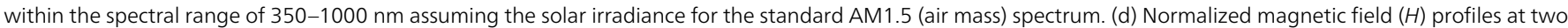

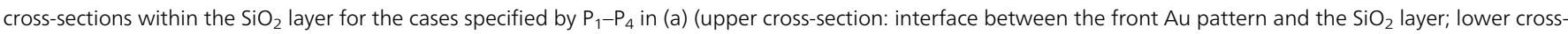
section: interface between the $\mathrm{SiO}_{2}$ layer and the gold back reflector). 
increases. The absorption peak at $\mathrm{P}_{2}(\lambda=590 \mathrm{~nm})$ is substantially influenced by $h_{\mathrm{SiO}_{2}}$ and becomes weaker for $\mathrm{SiO}_{2}$ thicknesses away from $60 \mathrm{~nm}$. This peak red-shifts as $h_{\mathrm{SiO}_{2}}$ increases and is a result of a dipole-dipole interaction between the triangles and the gold back reflector. ${ }^{28}$ The field profiles in Fig. 3d $\left(\mathrm{P}_{2}\right)$ verify the contribution of both triangles and the back reflector to this resonance. The absorption event at $\mathrm{P}_{3}$ mainly originates from the localized surface plasmon (LSP) resonance of the individual tapered triangles. Our simulations of an isolated tapered triangle on a glass substrate reveal a dominant absorption peak for $\lambda$ close to $720 \mathrm{~nm}$ (see ESI, Fig. S3 $\dagger$ ) which complies with the observed resonance at $P_{3}$. The position of this absorption peak is only slightly influenced by $h_{\mathrm{SiO}_{2}}$ and the weak field enhancement on the back reflector confirms the dominant contribution of the individual triangles to the resonance (see Fig. $3 \mathrm{~d}\left(\mathrm{P}_{3}\right)$ ). Absorption at $\mathrm{P}_{4}$ originates from the presence of the tips where energy is accumulated and dissipated. This is evident from the strong field enhancement at the tips shown in Fig. 3d $\left(\mathrm{P}_{4}\right)$.

Further evidence for the above-mentioned attributes is given in Fig. 3b, where calculated absorption spectra are plotted as a function of $R_{\text {bead }}$. Here, by maintaining the ratio $\Delta R / R_{\text {bead }}$ constant, we are able to keep the tip angle, $2 \theta$, constant and equal to $18.6^{\circ}$. Other geometrical values are $h_{\mathrm{SiO}_{2}}=60 \mathrm{~nm}$ and $h_{\mathrm{Au}, \mathrm{FP}}=$ $100 \mathrm{~nm}$. The two eye-guiding lines show the variation of the position of the resonances corresponding to $\mathrm{P}_{3}$ and $\mathrm{P}_{4}$ (see Fig. 3a). The resonance corresponding to $\mathrm{P}_{3}$ (dotted line) redshifts as $R_{\text {bead }}$ increases. This is in agreement with the expected red-shift of the LSP resonance of the triangles as their size (or equivalently $R_{\text {bead }}$ ) increases. ${ }^{29}$ On the other hand, the invariance of $2 \theta$ ensures a practically unchanged spectral position of the absorption peak corresponding to $\mathrm{P}_{4}$ (dashed-dotted line), although $R_{\text {bead }}$ and 2 d (see Fig. $1 \mathrm{~b}$ ) vary over a large range. As expected from previous studies, ${ }^{30}$ the focusing of the electromagnetic energy at the end of tapered tips is sensitive only to the tip opening angle. The spectral position of this absorption peak determines the upper cut-off of the absorption band and as seen in Fig. $2 b$ can be greatly increased by decreasing the tip angle, $2 \theta$.

In order to assess the performance of our concept in absorbing the solar spectrum, we calculate the total absorption as

$$
A_{\mathrm{tot}}=\frac{\int_{350 \mathrm{~nm}}^{1000 \mathrm{~nm}} A(\lambda) I(\lambda) \mathrm{d} \lambda}{\int_{350 \mathrm{~nm}}^{1000 \mathrm{~nm}} I(\lambda) \mathrm{d} \lambda}
$$

where $A(\lambda)$ and $I(\lambda)$ are the absorption of the structure and the solar irradiance for the standard AM1.5 (air mass) spectrum, respectively. In calculating the above absorption we consider the spectral range of 350-1000 nm, within which we performed measurements and simulations. The discarded part contains around $25 \%$ of the total solar energy. The contour plot in Fig. 3c shows the simulated total absorption as a function of thickness of the $\mathrm{SiO}_{2}$ layer, $h_{\mathrm{SiO}_{2}}$, and the size of the beads used for NSL, $R_{\text {bead }}$. In these simulations we used a constant value for $\Delta R / R_{\text {bead }}\left(=0.0133\right.$, corresponding to $\Delta R=2 \mathrm{~nm}$ at $R_{\text {bead }}=$ $150 \mathrm{~nm}$ ), to ensure a constant angle at the tips of the triangles,
20. Based on the above-mentioned studies, this assumption ensures almost the same upper cut-off wavelength of the absorption band for all the cases. The optimal geometry with $R_{\text {bead }}=200 \mathrm{~nm}$ and $h_{\mathrm{SiO}_{2}}=45 \mathrm{~nm}$ leads to a total absorption of $84 \%$. As explained before (see Fig. $2 \mathrm{~b}$ ), smaller values for $\Delta R$ can lead to substantially broader absorption spectra and therefore increase the total absorption. Furthermore, Fig. 3c shows that the total absorption around the optimum is quite insensitive to $h_{\mathrm{SiO}_{2}}$ and $R_{\text {bead }}$, making the performance of such a device highly tolerant to fabrication imperfections.

\section{Conclusions}

In conclusion, exploiting a combination of different plasmonic absorption phenomena in an array of tapered triangles integrated in an MIM configuration, we introduced a novel ultrabroadband plasmonic absorber with average absorption $88 \%$ within the spectral range of 380-980 nm. In addition, this absorber is insensitive to both light polarization and angle of incidence. While aspects of light absorption by arrays of tapered triangles have been studied before, our results contain significant novelty based on exploiting simultaneously four distinct absorption phenomena (each of different nature) two of which are accessible only through the MIM configuration. By suitably selecting a host of geometrical dimensions, we managed to harvest the collective benefit of these absorption events and pieced together a continuous absorption band. Our simulations show that one could go even beyond these high values, in terms of broadness and total absorption, by fine-tuning the geometrical parameters, specifically the tip regions of the tapered triangles in the pattern. Importantly, in contrast to previous successful designs, the fabrication of our concept is based on NSL which is a low-cost and easily accessible method. ${ }^{21,23}$ Although monocrystallinity is not a stringent requirement in our design, achieving large defect-free crystals with NSL remains a challenge. This bottleneck has been addressed in recent studies ${ }^{31,32}$ and significant improvements are foreseen. To this end, and based on the nano-scale thickness $(260 \mathrm{~nm})$ of our design, we envision the up-scaling of the absorber on flexible substrates even in a roll-to-roll manner. Being ultra-thin, the absorber can efficiently and rapidly elevate the temperature within a nano-scale layer, which can be exploited in various applications such as micro-fluidics, ${ }^{33}$ solar thermoelectrics ${ }^{4,9,34}$ and high temperature chemical reactions. ${ }^{35}$ In addition, efficiently absorbing the extremely broad solar light and concentrating it at the very end of the tips renders the present concept an interesting candidate for enhancing solar-based photochemistry and improving the efficiency of solid-state devices. ${ }^{5-8}$

\section{References}

1 H. A. Atwater and A. Polman, Nat. Mater., 2010, 9, 205-213.

2 J. V. van de Groep, P. Spinelli and A. Polman, Nano Lett., 2012, 12, 3138-3144.

3 C. H. Wu, B. Neuner, J. John, A. Milder, B. Zollars, S. Savoy and G. Shvets, J. Opt., 2012, 14, 024005. 
4 Y. J. Xiong, R. Long, D. Liu, X. L. Zhong, C. M. Wang, Z. Y. Li and Y. Xie, Nanoscale, 2012, 4, 4416-4420.

5 S. C. Warren and E. Thimsen, Energy Environ. Sci., 2012, 5, 5133-5146.

6 S. Linic, P. Christopher and D. B. Ingram, Nat. Mater., 2011, 10, 911-921.

7 F. M. Wang and N. A. Melosh, Nano Lett., 2011, 11, 5426-5430.

8 M. W. Knight, H. Sobhani, P. Nordlander and N. J. Halas, Science, 2011, 332, 702-704.

9 D. Kraemer, B. Poudel, H. P. Feng, J. C. Caylor, B. Yu, X. Yan, Y. Ma, X. W. Wang, D. Z. Wang, A. Muto, K. McEnaney, M. Chiesa, Z. F. Ren and G. Chen, Nat. Mater., 2011, 10, 532-538.

10 A. Moreau, C. Ciraci, J. J. Mock, R. T. Hill, Q. Wang, B. J. Wiley, A. Chilkoti and D. R. Smith, Nature, 2012, 492, 86-89.

11 M. K. Hedayati, M. Javaherirahim, B. Mozooni, R. Abdelaziz, A. Tavassolizadeh, V. S. K. Chakravadhanula, V. Zaporojtchenko, T. Strunkus, F. Faupel and M. Elbahri, Adv. Mater., 2011, 23, 5410-5414.

12 M. Elbahri, S. Homaeigohar, R. Abdelaziz, T. Dai, R. Khalil and A. U. Zillohu, Adv. Funct. Mater., 2012, 22, 4771-4777.

13 A. De Luca, N. Depalo, E. Fanizza, M. Striccoli, M. L. Curri, M. Infusino, A. R. Rashed, M. La Deda and G. Strangi, Nanoscale, 2013, 5, 6097-6105.

14 K. Aydin, V. E. Ferry, R. M. Briggs and H. A. Atwater, Nat. Commun., 2011, 2, 517.

15 M. G. Nielsen, A. Pors, O. Albrektsen and S. I. Bozhevolnyi, Opt. Express, 2012, 20, 13311-13319.

16 A. Aubry, D. Y. Lei, A. I. Fernandez-Dominguez, Y. Sonnefraud, S. A. Maier and J. B. Pendry, Nano Lett., 2010, 10, 2574-2579.

17 J. B. Pendry, A. Aubry, D. R. Smith and S. A. Maier, Science, 2012, 337, 549-552.

18 E. E. Narimanov and A. V. Kildishev, Appl. Phys. Lett., 2009, 95, 041106.
19 T. Sondergaard, S. M. Novikov, T. Holmgaard, R. L. Eriksen, J. Beermann, Z. H. Han, K. Pedersen and S. I. Bozhevolnyi, Nat. Commun., 2012, 3, 969.

20 T. Sondergaard and S. I. Bozhevolnyi, New J. Phys., 2013, 15, 013034.

21 J. C. Hulteen and R. P. Vanduyne, J. Vac. Sci. Technol., A, 1995, 13, 1553-1558.

22 B. J. Y. Tan, C. H. Sow, T. S. Koh, K. C. Chin, A. T. S. Wee and C. K. Ong, J. Phys. Chem. B, 2005, 109, 11100-11109.

23 X. Y. Zhang, A. V. Whitney, J. Zhao, E. M. Hicks and R. P. Van Duyne, J. Nanosci. Nanotechnol., 2006, 6, 1920-1934.

24 S. R. Ye, A. L. Routzahn and R. L. Carroll, Langmuir, 2011, 27, 13806-13812.

25 E. D. Palik and G. Ghosh, Handbook of optical constants of solids, Academic Press, San Diego, 1998.

26 N. Liu, M. Mesch, T. Weiss, M. Hentschel and H. Giessen, Nano Lett., 2010, 10, 2342-2348.

27 Y. Z. Chu and K. B. Crozier, Opt. Lett., 2009, 34, 244-246.

28 W. Rechberger, A. Hohenau, A. Leitner, J. R. Krenn, B. Lamprecht and F. R. Aussenegg, Opt. Commun., 2003, 220, 137-141.

29 K. L. Kelly, E. Coronado, L. L. Zhao and G. C. Schatz, J. Phys. Chem. B, 2003, 107, 668-677.

30 Y. Luo, J. B. Pendry and A. Aubry, Nano Lett., 2010, 10, 41864191.

31 G. D. Moon, T. I. Lee, B. Kim, G. Chae, J. Kim, S. Kim, J. M. Myoung and U. Jeong, ACS Nano, 2011, 5, 8600-8612.

32 C. C. Ho, P. Y. Chen, K. H. Lin, W. T. Juan and W. L. Lee, ACS Appl. Mater. Interfaces, 2011, 3, 204-208.

33 J. S. Donner, G. Baffou, D. McCloskey and R. Quidant, ACS Nano, 2011, 5, 5457-5462.

34 K. Moth-Poulsen, D. Coso, K. Borjesson, N. Vinokurov, S. K. Meier, A. Majumdar, K. P. C. Vollhardt and R. A. Segalman, Energy Environ. Sci., 2012, 5, 8534-8537.

35 C. Fasciani, C. J. B. Alejo, M. Grenier, J. C. Netto-Ferreira and J. C. Scaiano, Org. Lett., 2011, 13, 204-207. 